\title{
Bildung.Macht.Diversität - ein verschlungenes Feld
}

Ulla Klingovsky, Serena 0. Dankwa, Sarah-Mee Filep und Georges Pfruender

Die Begriffe Bildung und Diversität eröffnen den Blick auf ein machtvoll verschlungenes Feld, das von Akteur*innen unterschiedlicher wissenschaftlicher Disziplinen und gesellschaftlicher Handlungspraxen bearbeitet wird. Dabei schwankt die Beschäftigung mit Fragen zu Diversität im Kontext von Bildung stets zwischen Begeisterung und Problemdiagnose. Auf der einen Seite werden die vielfältigen Potenziale von (scheinbar individuellen) Besonderheiten beschworen, auf der anderen Seite werden Handlungsweisen, die von einer unhinterfragten Norm abweichen, problematisiert und dies häufig mit Bezug auf eine (vermeintliche) Gruppenzugehörigkeit. Sowohl die freudvolle Affirmation von Diversität wie deren Abwehr gehen allerdings von einer wesenhaften, kategorisierbaren Verschiedenheit, von einer identifizierbaren "Andersheit« aus, die entweder als etwas Potentes gefeiert oder als etwas Störendes abgewertet und zum Verschwinden gebracht wird. In beiden Fällen bleibt das machtkritische Potenzial von Diversität ungenutzt und zugleich bleiben die gewaltvollen Praktiken der Zuschreibung, Adressierung und Kategorisierung von Verschiedenheit unsichtbar - nicht zuletzt in Bildungskontexten.

Bildung und Bildungsinstitutionen sind auf vielfältige Weise in gesellschaftliche Machtverhältnisse involviert. Versteht man Bildung auf der einen Seite als Prozess, lässt sich behaupten, dass sie Diskriminierungs- und Differenzerfahrungen durch die Hervorbringung neuer Deutungs- und Verhaltensmuster zu verarbeiten hilft. Auf der anderen Seite sind diese Muster der Verarbeitung Produkt und Resultat sozialer Unterscheidungspraxen, entlang derer machtvolle Normalitätsordnungen hervorgebracht werden. Bildung findet damit stets in einer Gesellschaft machtvoller Differenzen statt, die zu bedeutungsvollen Unterscheidungen werden. Machtverhältnisse bilden schließlich die Klammer um Fragen von Diversität in Bildungskontexten. Denn sowohl Bildung als auch Diversität lassen sich nur im Kontext gesellschaftlicher Machtverhältnisse verstehen und angemessen bearbeiten. Der hier vorliegende Sammelband greift verschiedene relevant gesetzte Differenzlinien auf. Dabei orientiert er sich nicht an disziplinären, professionellen oder aktivistischen Grenzen, sondern erkundet ein weites, unübersichtliches Terrain, auf dem sich gesellschaftliche Auseinandersetzungen um ungerechte Ver- 
hältnisse, institutionelle Diskriminierung und verdeckte Formen der Ausgrenzung entzünden.

Dieser Band begnügt sich nicht mit der Vorstellung, dass eine vielfältigere und vielstimmigere Gesellschaft möglich und ein kritischer Zugang zu Diversität erlernbar ist. Ein erster Schritt besteht darin, zu erkennen, wie Machtverhältnisse historisch gewachsen und in unsere Denk- und Handlungsweisen eingeschrieben sind und wie ungleich verteilte Ressourcen Differenzen herstellen. Doch in Anlehnung an das Konzept der Critical Diversity Literacy verändert allein das »Lesen« von Privilegien und Diskriminierungen die Welt noch nicht. Um einen Prozess hin $\mathrm{zu}$ einer weniger gewaltvollen Gesellschaft anzustoßen, müssen wir auch Schreiben lernen. Wie dieser Schreibprozess aussehen soll, ob die alten Skripts zuerst einmal zerrissen werden müssen, ob sie verlernt werden können oder ob sich Vorhandenes über- und umschreiben lässt und wie wir eine sozial gerechtere, eine freiere oder eine gänzlich neue Welt anstreben oder imaginieren, das bearbeiten die Beiträge in diesem Band aus verschiedenen Perspektiven und mit vielfältigen Werkzeugen.

Der gemeinsame Ausgangspunkt ist die Hochschule - eine Welt, die von jahrhundertalten Hierarchien und normativen Wissensordnungen, von Leistungsdruck und Elitarismus geprägt ist. Zugleich ist die Hochschule auch ein Ort für Problematisierungen. Durch ihr institutionalisiertes Recht auf Kritik werden in diesem Sammelband gegebene Verhältnisse befragt und neue Denkwege hervorgebracht, welche die Institution von innen heraus kritisieren und $\mathrm{zu}$ bewegen versuchen.

\section{Entstehungskontexte}

Die Entstehung eines Sammelbandes umfasst stets vielfältige Anfänge. Eine bedeutsame Inspirationsquelle für den vorliegenden Band ist der transnationale Forschungsaustausch der Professuren für Kulturvermittlung/Theaterpädagogik und Erwachsenenbildung/Weiterbildung an der Fachhochschule Nordwestschweiz (FHNW) mit dem Wits Centre of Diversity Studies an der University of the Witswatersrand in Johannesburg. Die Gründerin und Leiterin dieses interdisziplinären Zentrums, Melissa Steyn, analysiert mit ihrem Team den Kontext und die Geschichte des Prozesses der Post-Apartheid-Nationenbildung. Dabei bearbeitet sie verschiedene Facetten der tiefen gesellschaftlichen Zerrissenheit, in der ein Umgang mit den historischen, sozialen und kulturellen Verwerfungen, Ausschlüssen und Ungerechtigkeiten gefunden werden muss. Diese Auseinandersetzungen bilden die Hintergrundfolie für die Forschungs- und Entwicklungsarbeit zum Thema Critical Diversity Literacy, die sich in Steyns gleichnamigen Konzept entfalten (2015). 
In ihrer Auseinandersetzung mit theoretischen Konzeptionen wie dem politischen Antirassismus ${ }^{1}$, der Critical Race Theory (vgl. Crenshaw et al. 1995) und der Critical Whiteness (vgl. hooks 1990) entwirft Steyn Möglichkeiten einer produktiven Bearbeitung von Fragen der Diversität für gesellschaftliche (Bildungs-)Institutionen. Dabei dekonstruiert sie die Epistemologie belohnter Ignoranz (»epistemology of ignorance«) als Routine des »aktiven Vergessens der Anderen« (Mills 1997: 18). Diese Routine identifiziert sie als strukturell verankerte politische Position im Sinne einer perpetuierten Uninformiertheit, die keine Peinlichkeit auslöst, weil auf ihrer Grundlage die eigenen Privilegien behauptet und legitimiert werden (vgl. Steyn 2015).

Mit ihren machtanalytischen Forschungsarbeiten zu Critical Diversity Literacy eröffnet Melissa Steyn die theoretische und analytische Grundlage für das transnationale Forschungs- und Entwicklungsprojekt »Critical Diversity Literacy arts \& further education«, mit dem - informiert über Theorien und Praxen der Künste - bildungstheoretisch fundierte (Weiter-)Bildungsräume konzipiert und eröffnet werden. Diese sensibilisieren für soziale und kulturelle Machtverhältnisse, $\mathrm{Zu}$ schreibungen und normative Setzungen gegenüber "Anderen « und für die damit einhergehenden Prozesse der Ein- und Ausgrenzung.

Im Herbst 2016 begann die gemeinsam mit Melissa Steyn und ihrem Team initiierte Projektkonstellation Critical Diversity Literacy arts \& further education Gestalt anzunehmen. Dabei wurde das Konzept der Kontaktzone ${ }^{2}$ als Bezugspunkt der $\mathrm{zu}$ entwickelnden Weiterbildungskonzeption bestimmt und zugleich als Leitprinzip der gemeinsamen Forschungs- und Entwicklungsarbeit beschrieben. Die gemeinsame Kritik an einem unproblematisierten Zugang zu »interkulturellem Dia-

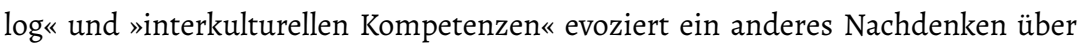
die (Un-)Möglichkeiten dieser transkulturellen Begegnung. Geteilt wird die Annahme, dass kein unkritischer Austausch unter gleichberechtigten Parteien unterstellt werden soll, da der Austausch unter den gegebenen hegemonial strukturierten,

Das Konzept des politischen Antirassismus unterscheidet sich analytisch, aber auch strategisch von anderen, psychologisierenden oder moralisierenden Konzepten. Rassismus wird nicht als punktuelles Phänomen oder individuelles Fehlverhalten verstanden, sondern als Struktur, die hegemoniale Machverhältnisse, Diskurse und Praktiken trägt. Strategisch setzt politischer Antirassismus auf die Sichtbarmachung rassistischer Strukturen auf allen gesellschaftlichen Ebenen und auf die Ermächtigung von marginalisierten oder diskriminierten Subjekten (vgl. Bratič 2012).

2 Kontaktzonen sind Formen kollektiv erstellter Räume, in denen unterschiedliche soziale und kulturelle Positionen aufeinandertreffen und eine räumliche Ordnung unter gegebenen Machtverhältnissen performativ immer wieder neu hervorgebracht wird (vgl. Pratt 1996: 6f.) Das Konzept »contact zone« wird in der feministischen Theorie der Critical Race Theory und den Colonial Studies verwendet (vgl. Sternfeld 2013). 
institutionalisierten sowie in historisch kolonialen und aktuell neokolonialen Verhältnissen stattfindet, die ein etabliertes und sich permanent reproduzierendes Machtungleichgewicht provozieren. Darüber hinaus soll die ungleiche Verteilung von Ressourcen wie Geld, Definitionsmacht bzw. die unterschiedlich gute Kapitalisierbarkeit verschiedener Wissensbestände kontinuierlich benannt und sichtbar gehalten werden. Für transnationale Suchbewegungen scheint der Begriff der Kontaktzone besonders geeignet. Denn es geht zunächst darum, die historisch und kontextuell je unterschiedlich gewordenen Vorstellungen davon, was wichtige und im Sinne einer Critical Diversity Literacy geeignete theoretische Verhältnisbestimmungen und konzeptionelle Bearbeitungsmodi wären, gemeinsam in Verhandlung und damit in Bewegung zu bringen.

Die Suche nach einem konkreten Bearbeitungsfeld in der Schweiz fokussierte relativ rasch auf die Fachhochschule als einer akademischen Institution, die strukturell und konzeptionell einen engen und ausgewiesenen Bezug zu konkreten gesellschaftlichen Praxisfeldern aufweist. Die programmatische Handlungsorientierung des Konzepts der Critical Diversity Literacy sollte hier nicht nur für die Analyse und Bearbeitung impliziter Normalitäts- und Wissensordnungen der Hochschule fruchtbar gemacht werden, sondern mit Blick auf die dringende Notwendigkeit auch in deren jeweiligen Praxisfeldern und gesellschaftlichen Bezugsräumen grundlegende Veränderungsprozesse evozieren. Die Fachhochschulen in der Schweiz weisen ein hohes Potenzial für diese Veränderungsprozesse auf, da sie im Zuge der Vereinheitlichung und Akademisierung von höherer Berufsbildung an Bedeutung gewonnen haben und nach wie vor breiter zugänglich und hinsichtlich der Klassenzughörigkeit auf allen Ebenen diverser sind als die Universitäten.

Die Bildungskonzeption Critical Diversity Literacy zielt auf die Ermöglichung einer kritisch-performativen Lektürepraxis zu Fragen von Diversität, d.h. auf die Analyse bestehender Machtverhältnisse und die Imagination von deren Transformation. Im Zentrum dieser Entwicklungsarbeit steht also keine hochschuldidaktische Maßnahme zur Schulung und Vermittlung »interkultureller Kompetenzen«, sondern eine kollektive Suchbewegung und das gemeinsame Erstellen von Kontaktzonen (vgl. Sternfeld 2013). Die zentrale Herausforderung besteht in der Konzeption und Gestaltung einer spezifischen Räumlichkeit, in der vielfältige Interaktionen zwischen Menschen, die unterschiedlich in die Machtstrukturen der Hochschule involviert sind, möglich werden. In diesen konkreten Begegnungen werden jene machtvollen Verhältnisse und Strukturen einer kritisch-performativen Lektürepraxis unterzogen, mit denen die Hochschule Zugangsberechtigungen erteilt und Zugänge versperrt, Berufsbiografien anbahnt und Aufstiegsambitionen systematisch auskühlt, intersektionale Diskriminierungen prozessiert und soziale Chancen und Privilegien zu- und verteilt. 


\section{Der Raum als Weiterbildungsatelier}

»Weiterbildungsatelier« ist der Begriff für diese spezifische Räumlichkeit, in der sich kollektive Suchbewegungen entfalten, Begegnungen stattfinden, Kontaktzonen installiert und Verhandlungen eröffnet werden. Die Gestaltung dieser Ateliers schafft die Gelingensbedingungen für eine kritische-performative Lektürepraxis. Die Teilnehmenden der Weiterbildungsateliers sind temporär freigestellt von den Zwängen und Verpflichtungen ihres Alltags und finden sich in einem Raum, in dem Verunsicherungen und Begegnungen mit offenem Ausgang erprobt werden können. In der Handlungsentlastetheit eines solchen Raums eröffnet sich die Möglichkeit, $\mathrm{zu}$ jenen Verfahren, Mechanismen, Routinen und Konventionen in Distanz zu treten, in denen sich zeigt, auf welche Weise gesellschaftliche Machtverhältnisse in gesellschaftliche Institutionen eingeschrieben sind. Solche Ateliers konzipierten wir an der FHNW zusammen mit Kolleg*innen, die als »critical friends« eingeladen waren, die Szenarien, Formate und Weiterbildungskonstellationen anzureichern und für die verschiedenen Arbeitskontexte fruchtbar zu machen. Lesen lernen beginnt hier mit der Distanzierung und Einklammerung des institutionell Vertrauten und mündet in die Be- und Erforschung der Hochschule mitsamt ihren machtvollen Verfahren und Mechanismen.

In der hier vorgenommenen machtkritischen Analyse von Fragen der Diversität erweist sich die Hochschule keineswegs als »unschuldiger« Ort der Vermittlung gleich-gültiger Wissensbestände, sondern als ein machtvolles Instrument gesellschaftlicher Verteilungskämpfe. Hier werden Bildungsprozesse ermöglicht und zugleich als ein zentrales Mittel eingesetzt, um Abgrenzungen und Marginalisierungen vorzunehmen respektive unterschiedliche Platzierungen von Menschen zu legitimieren (vgl. Mecheril/Klingler 2010: 93). Von hier aus kann - inspiriert von Theorien und Praxen der Künste - eine Bewegung der Dezentrierung ihren Anfang nehmen, die zu Probehandlungen und zum Experimentieren mit alternativen Entwürfen, Konzepten und Imaginationen einlädt. Konkret wurde ein Theaterraum an der FHNW zu dem Ort, an dem diese Ateliers stattfinden und zu Inseln im Hochschulalltag werden konnten. Diese Räumlichkeit entwickelte sich zur Herzschlagader des Gesamtprojekts Critical Diversity Literacy arts \& further education, aus dem heraus sich neue Verbindungen und Ausgangspunkte für weitere Bearbeitungen an der Hochschule und darüber hinaus bilden konnten.

Neben der Notwendigkeit von derart verorteten (Denk-)Räumen können Prozesse der Verunsicherung und, vielleicht, des Verlernens nur angestoßen werden, wenn zugleich die Begegnung mit einem »anderen«, nicht-hegemonialem Wissen ermöglicht wird (vgl. Wagner und Castro Varela in diesem Band). Eine zentrale Aufgabe ist es dabei, für die Expert*innen mit einem "anderen« Wissen adäquate Bedingungen für die institutionalisierte Zusammenarbeit - von der Konzeption und Vorbereitung bis hin zur Gestaltung und Moderation der Ateliers - zu schaf- 
fen. Hierfür sind nicht nur die notwendigen Ressourcen zu sichern, um die Expertise beratender und unterstützender Akteur*innen und Aktivist"innen honorieren zu können, sondern auch die Zugänglichkeit des Hochschulraums hinsichtlich seiner intellektuellen, affektiven und körperlichen Barrieren kritisch zu überprüfen. Darüber hinaus gilt es, in unterschiedliche Distanzen zu den eigenen institutionellen Gewohnheiten und Routinen zu treten und sich in unbekanntes Terrain zu begeben - ein Terrain, das sich immer auch in unbefragten Aspekten des eigenen Gewordenseins lokalisieren lässt. So werden in dem Weiterbildungsatelier »Undoing Identities. Narrationen des Selbst « vielfältige Marginalisierungs- und Privilegierungserfahrungen entlang biografischer Geschichten aufgearbeitet, um sie im Kontext der strukturell ungleichen Verteilung von Ressourcen in Bewegung bringen zu können.

Im allmählichen Entstehungsprozess der verschiedenen Ateliers zeichnet sich eine Bewegung hin zur Bearbeitung konkreter Diskriminierungs- und Differenzachsen $a b$, welche die machtvollen Kontexte und Funktionsweisen von Differenz ergründet und befragt. Unter dem Titel »Prozesse der Rassifizierung - Dechiffrierung durch Zeitungstheater « beleuchtet eines der Ateliers rassistisch strukturierte Macht- und Herrschaftsverhältnisse und ihre vielfältigen Ausprägungen an der Hochschule. Mit dem performativen Zugang des Zeitungstheaters von Augusto Boal werden an der Hochschule alltäglich erlebbare Alltagsrassismen ebenso problematisiert wie die Mechanismen in der Hochschule, mit denen rassifizierte $\mathrm{Zu}$ gangsbarrieren unsichtbar gehalten werden. Nicht zuletzt steht auf der Grundlage von Texten, in denen sich Forschende of Colour mit weißen Privilegien beschäftigen, die Frage im Zentrum, wie sich akademisches Wissen konstelliert und wessen Wissen in der Hochschule gelernt und gewusst werden soll (vgl. Heinemann/Keser in diesem Band).

Das Atelier »un.Sichtbare ver.Hinderung - Perspektivwechsel und Performance« stellt die Befragung der Normen und Normierungen an der Hochschule im Kontext von Disability in den Mittelpunkt. Hier sind es inszenierte Perspektivwechsel, die verschiedene Zugangsbarrieren und Ver-Hinderungsstrukturen an der Hochschule sichtbar machen und in Verhandlung bringen. Als ein konkreter Schritt hin zur Verschiebung der eigenen Perspektive fand ein erstes Vorbereitungstreffen dieses Ateliers mit dem Experten und Performer Edwin Ramirez (www.rollingeddie.ch) außerhalb des Hochschulraums im Les Complices (www.lescomplices.ch) statt, einem kollektiven Projekt- und Kulturraum, der von Queer of Colour Aktivist*innen betrieben wird. Um das Wissen und die Expertise von Menschen zu finden, deren Zugang zur Hochschule durch ableistische Strukturen erschwert und verhindert wird und deren Zeitlichkeit der unmarkierten Leistungsund Mobilitätsnorm nicht entsprechen kann (z.B. durch eine aufwendige Suche nach rollstuhlgängigen Trams und Zügen), ist die aufmerksame Bereitschaft gefordert, sich von den hegemonialen Zentren (in diesem Fall von der Hochschule) 
weg zu bewegen. Das gemeinsame Denken und Arbeiten in dieser dezentrierten Räumlichkeit war für die Konzeption und Konstellierung des thematisch einschlägigen Ateliers wegweisend.

Diese Ateliers fokussieren thematisch auf einzelne Achsen der Differenz, ohne dabei die Machtverhältnisse zu vergessen, die der Hervorbringung relevanter Differenzkategorien zugrunde liegen (vgl. Meyer/Purtschert). Vor diesem Hintergrund einer intersektionalen Analyseperspektive werden in den Ateliers Veränderungsnotwendigkeiten auf drei hochschulischen Handlungsebenen sichtbar und bearbeitbar. Auf der Makroebene geht es um Fragen des Zugangs zur respektive der Öffnung der Hochschule, auf der Mesoebene um Fragen der curricularen Gestaltung von Studiengängen und -modulen und schließlich auf der Mikroebene um Fragen einer diversitätsorientierten Gestaltung interaktiver Lehr- und Lernsituationen mit Studierenden. Insgesamt visiert eine kritisch-performative Lektürepraxis ein (politisches) Engagement für die Zerstreuung, Transformation und Verflüssigung der diesen Strukturen zugrundeliegenden Machtverhältnisse und Orientierungen im Sinne der Entwicklung anderer, vielleicht gerechterer gesellschaftlicher (Bildungs-)Institutionen.

Die Ateliers wurden in dieser Form als Prototypen konstelliert, mit deren Hilfe die analytische Reflexion verschiedener Aspekte von Critical Diversity Literacy kontinuierlich vertieft und geschärft werden kann. Obgleich der Wunsch nach klaren Orientierungen und einer Reduktion von Komplexität in Anbetracht der vielschichtigen und spannungsreichen Verhältnisse von Bildung, Macht und Diversität naheliegend scheint, entzieht sich eine kritisch-performative Lektürepraxis jedweder Vereindeutigung selbst dann, wenn diese in kritischer Absicht erfolgt. Sie eröffnet viel eher einen analytischen Blick auf das noch Ungesicherte und Ungeklärte in der Beschäftigung mit Fragen von Diversität und offenbart Verunsicherungen, Lücken und Unklarheiten bis hin zu Paradoxien und Widersprüchen, die sich möglicherweise nicht auflösen werden. Im Folgenden sollen zum einen einige dieser Zusammenhänge beleuchtet werden, die kaum eindeutig geklärt werden können, da sie bei genauerer Analyse ihre Mehrdeutigkeit offenbaren, und zum anderen sollen Prozesse aufgedeckt werden, in denen sich nicht notwendigerweise gewünschte Intentionen, sondern unerwünschte Nebenwirkungen realisieren.

\section{Von Paradoxien und Widersprüchen}

In der Konzeption und Gestaltung der Weiterbildungsateliers erkannten wir alsbald die Schwierigkeit, die Konturen und Mechanismen unterschiedlicher Differenz- und Diskriminierungsachsen zu erforschen, d.h. die Notwendigkeit, sich grundlegende historische Kenntnisse über die spezifischen Wirkungsweisen von Sexismus, Ableismus oder Rassismus und deren Widerstandsbewegungen 
aneignen zu müssen, um überhaupt intersektional denken lernen zu können und zugleich mit der Rede von Diversität bereits einen Gesamtkontext überblicken zu wollen. Die präzise Auseinandersetzung mit unterschiedlichen Privilegien und Diskriminierungskontexten und der dazugehörigen Expertisen ist von zentraler Bedeutung, wenn es zu verhindern gilt, dass Diversity gleichmachend und entpolitisierend wirkt. Wie Kimberlé Crenshaw (1989) aufzeigt, bergen Gleichstellungsund Antidiskriminierungspolitiken stets die Gefahr, von oben herab zu agieren und dadurch nur den privilegiertesten Menschen innerhalb einer marginalisierten Gruppe und nur dem Wissen, das dem eigenen am ähnlichsten ist, institutionelle Zugänge zu ermöglichen.

Wenn wir einen Raum diskriminierungskritisch umgestalten und transformieren wollen, verstricken wir uns auch an der Hochschule unweigerlich in Zugangsparadoxien. Solche entstehen etwa dann, wenn sowohl der Assistenzhund einer blinden Person als auch die Hundeallergikerin in einem Raum Platz finden sollen oder wenn ein Raum sowohl für Menschen mit Konzentrationsschwierigkeiten als auch für Eltern mit ihren Kleinkindern zugänglich sein soll. Aktivist*innen ${ }^{3}$ mit Behinderungen ermutigen dazu, sich diesen Zugangsparadoxien zu stellen und die dazugehörigen Aushandlungsprozesse, gerade auch im Hochschulraum, als kreative Ver-Lernprozesse zu begreifen, statt in der Komplexität der Fragen zu erstarren. Im Wissen darum, dass Imperfektion zum Leben gehört, haben Menschen mit Behinderungen gelernt, unaufgeregt und improvisierend mit konkurrenzierenden Zugangsbedürfnissen umzugehen. Dabei haben sie eine Ästhetik der Zugänglichkeit entworfen, die nicht beim ärgerlichen Abarbeiten dieser Hürden stehen bleibt, sondern diese wahrnehmbar macht und die Schönheit dieser Prozesse erkennt und weiterentwickelt (vgl. Mühlemann in diesem Band).

Der Anspruch, möglichst vielen Menschen Zugang zu kritischem Wissen in der Akademie zu verschaffen, führt unter den Bedingungen des zunehmend ungesicherten, wettbewerbsorientierten und drittmittelfinanzierten Arbeitens an den Hochschulen zu widersprüchlichen Konstellationen. Ein konkretes Beispiel hierfür offenbart sich in der Frage der sozio-ökonomischen Zugänglichkeit dieses Sammelbandes, der am Ende einer mehrjährigen Projektfinanzierung erscheint. Es war unser Ziel, die privilegierte Verortung an einer Schweizer Hochschule bzw. deren Zugang zu nationalen Fördergeldern zu nutzen, um die Möglichkeit einer OpenAccess-Publikation zu prüfen. Gleichzeitig stellte sich heraus, dass wir zwar berechtigt sind, beim Schweizerischen Nationalfonds einen Antrag auf Publikationsförderung zu stellen, aber dass ein solcher mit dem dazugehörigen stattfand, ermutigten die Teilnehmenden, das Imperfekte als perfekt und zum Leben gehörend zu betrachten. 
Peer-Review-Verfahren den Prozess dahingehend verzögern würde, dass die Publikation erst lange nach Ablauf der befristeten Anstellungsverhältnisse von zwei der vier Herausgeber*innen erfolgen würde. Aufgrund unserer Lebensrealitäten und in Ermangelung von Langzeitperspektiven im neoliberalen Hochschulbetrieb Perspektiven, die sich mit den ökonomischen Notwendigkeiten und den Zeitlichkeiten unserer (queeren) Lebensentwürfe decken würden - entschieden wir uns gegen einen Antrag. Glücklicherweise konnten wir dank zusätzlicher Leistungen unsererseits doch eine OpenAccess-Publikation bei transcript erwirken, ohne dass der Verkaufspreis dadurch massiv in die Höhe geschnellt wäre. Der Widerspruch von prekären Anstellungsverhältnissen und dem Wunsch, ein wichtiges Buch über Diversität einem breiteren Publikum frei zugänglich zu machen, erforderte einiges an Kreativität und Geschick, um die notwendigen Ressourcen zu sichern - jene Kreativität, die zur Bedingung geworden ist oder schon immer war, um kritischem Wissen in der Akademie Gehör zu verschaffen.

Eine weitere Paradoxie, die uns in der Erarbeitung dieser Publikation umtrieb und darüber hinaus auf die notwendige Entwicklung einer kritischen Diversitätsgrammatik und eines Vokabulars verweist, die eine Diskussion über Privilegien und Diskriminierung an der Hochschule ermöglicht, betrifft die Gendergerechtigkeit. Das Resultat unserer Suche nach einer Sprache, die einerseits nicht-normierte Geschlechter wahrnehmbar macht und andererseits kompatibel ist mit den Screenreader- und Sprachausgabeprogrammen, die den Bildschirminhalt vorlesen und somit Menschen mit einer Sehbehinderung den Zugang erleichtern, ist uneindeutig geblieben. Eine neue Schreibweise, die als Alternative zu Unterstrich (Gendergap) oder Sternchen (Asterisk) verwendet wird, ist der Doppelpunkt. Wenn ein Sprachausgabeprogramm so eingestellt ist, dass Satz- und Sonderzeichen nicht vorgelesen werden, entsteht sowohl beim Doppelpunkt als auch bei Unterstrich und Sternchen eine Lücke. Diese gesprochene Lücke kommt dem Glottiverschluss nahe, der zunehmend auch im alltäglichen Sprachgebrauch Eingang findet, um Vielgeschlechtlichkeit in Bezeichnungen wie Pädagog*innen hörbar zu machen. Nun ist es aber so, dass verschiedene Sprachausgabeprogramme das Vorlesen von Satz- und Sonderzeichen unterschiedlich gut handhaben. Und noch unterschiedlicher sind die Menschen, die mit verschiedenen Sehbehinderungen leben, mit verschiedenen Bildungsprivilegien und Budgets für Sprachausgabeprogramme, aber auch mit vielfältigen Geschlechtsidentitäten. Entsprechend unterscheiden sich die Vorlieben und Bedürfnisse, was das Gendern anbelangt, so der blinde Aktivist und Inklusionsexperte Heiko Kunert. Er kritisiert die These, das Gender-Sternchen sei diskriminierend und argumentiert, dass auch sehende Leser*innen durch Unterstrich und Sternchen irritiert werden (sollen). Stattdessen plädiert er dafür, »den Druck auf Screenreader- und Sprachausgaben-Entwickler*innen zu erhöhen, damit in der Zukunft ein korrektes Vorlesen gegenderter Begriffe erfolgt«. 
Wir haben keinen Druck gemacht auf die Screenreader-Entwicklung. Und wir haben uns gegen den Doppelpunkt und für Unterstrich und Sternchen entschieden. Während diese Doppelspurigkeit unserem Bedürfnis, unterschiedliche Politiken des Genderns zuzulassen, Ausdruck verleiht, wären wir zugleich gerne der informellen Empfehlung des Schweizerischen Blinden- und Sehbehindertenverbands SBV nachgekommen, möglichst »einheitlich « zu gendern. ${ }^{4}$ Hier haben wir uns, zusammen mit den meisten Autor*innen dieses Bandes für das Sternchen entschieden, weil es, wie uns scheint, weniger abtrennend und binär wirkt als der Doppelpunkt (auch wenn es vielleicht tatsächlich den Doppelpunkt oder andere neue Satzzeichen braucht, um Gendervielfalt weiterhin sichtbar zu halten) und in der Hoffnung, dass das Sternchen weiterhin über sich hinausstrahle und Räume öffne für die vergeschlechtlichten und sexuellen Seinsweisen, die da waren, die da sind und die noch kommen werden. Wir sind uns bewusst, dass auch das Sternchen in den letzten Jahren inflationär zum Einsatz gekommen ist und damit einiges an imaginärer Kraft verloren hat. Wenn etwa hinter einem gegenderten Begriff wie »Frauen« ein Sternchen gesetzt wird als Hinweis darauf, dass alle, die sich mit dem Frausein identifizieren oder auseinandersetzen (mussten) in der Runde mitgemeint sind, ist das zwar gut gemeint, aber dennoch problematisch, wenn die Mitgemeinten nicht in die Problemstellung und Gestaltung der Runde miteinbezogen worden sind. ${ }^{5}$ Es geht also nicht nur um Symbolik, sondern vor allem auch um reale räumliche Anordnungen bzw. die Frage, ob sich besternte Menschen in einem Raum tatsächlich willkommen und sicher fühlen können oder nicht. Es gibt keine einfachen Auswege aus der Logik der binären Kategorisierungen. Wir stehen am Anfang der Diversifizierung und vielleicht der Auflösung von Geschlechterkategorien und diese Irritation soll und muss auch sprachlich sichtbar sein.

Paradoxien entstehen in der Sprache auch dann, wenn wir Gewalt thematisieren wollen, ohne diese verbal zu reproduzieren. Ein Ringen um eine zugleich zugängliche und dennoch nuanciert sorgsame Sprache begleitete insbesondere die Auseinandersetzungen mit Rassismus in diesem Sammelband. In dem Zusammenhang schreiben wir Schwarz groß, um nicht auf eine vermeintliche Hautpigmentierung, sondern auf Schwarz als Selbstbezeichnung und als politische Kategorie zu rekurrieren. Diese verweist auf das symbolische Kapital, das sich Menschen und Kollektive in Afrika und der Schwarzen Diaspora im Widerstand gegen Rassismus erkämpft haben. Einige Autor*innen des Bandes schreiben zusätzlich den Begriff weiß kursiv, um weiß als eine meist unmarkierte soziale Position von Menschen, die im Alltag keinen Rassismus erfahren, zu markieren. Wer als weiß zählt,

4 Gemäß Telefongespräch mit Rolf Roth (2.12.2020) vom SBV https://www.sbv-fsa.ch [15.3.2021].

5 Siehe Beitrag Stars und Sternchen von Hengameh Yaghoobifarah im Missy Magazin, https:// missy-magazine.de/blog/2018/05/11/stars-und-sternchen/ [15.3.2021]. 
ist dabei nicht abschließend definiert, sondern letztlich kontextabhängig. Diese Schreibweise, die auf Mythen, Masken und Subjekte (Eggers/Kilomba/Piesche/Arndt 2005), den ersten Sammelband zur Kritischen Weißseinsforschung in Deutschland, zurückgeht, hat im deutschsprachigen Raum dazu beitragen, dass weiß als soziale Zugehörigkeits- und politische Kategorie überhaupt benennbar wurde. Weitere Autor*innen wiederum haben sich bewusst dafür entschieden, weiß nicht kursiv zu schreiben, um den Begriff in keiner Weise zu besondern - eine Schreibweise, die auch dem Englischen entspricht.

Obwohl es gute Gründe gibt, den deutschen, biologistisch geprägten Begriff der »Rasse« nicht aus der Erinnerung zu entlassen, verwenden wir Race oder Rassifizierung, um auf den Konstruktionscharakter dieses Herrschaftsverhältnisses hinzuweisen. Im englischen Sprachraum, in dem der Begriff Race auch in seinem sozial konnotierten Plural existiert und die Kritik am kolonialrassistischen Erbe tiefer verankert und breiter rezipiert ist, fällt es leichter, Rassifizierung zu benennen, manchmal aber auch auf die Gefahr hin, dass die Gewalt, die bestimmten Begriffen innewohnt, heruntergespielt oder reproduziert wird. Als Herausgeber*innen sind wir deshalb darum bemüht, Race auch auf Englisch sparsam zu verwenden und kursiv zu schreiben. Und nicht zuletzt laufen Debatten um Rassismus und Intersektionalität allzu häufig Gefahr, sich in Argumenten und Gegenargumenten rund um Sprache und Repräsentation zu erschöpfen, um den Preis, dass die schwierige und zugleich dringend notwendige Bekämpfung institutionalisierter Gewalt und transnationaler Ausbeutungsverhältnisse und der damit einhergehenden (rassifizierten) Subalternisierung mitunter in den Hintergrund rückt.

\section{Zu den Beiträgen des Bandes}

Mit den Beiträgen in diesem Band eröffnen wir die Diskussion zu den genannten Paradoxien und vielfältigen Widersprüchen in der Auseinandersetzung mit Fragen von Diversität im Hochschulraum. Hierfür werden die verschiedenen in dieser Einleitung bereits aufgefächerten Differenzlinien im Kontext der Hochschule vertieft bearbeitet. Die Hochschule wird dabei als Spiegel und Manifestation gesellschaftlicher Verhältnisse, aber auch als Motor notwendiger Veränderungsprozesse beleuchtet. Im Zentrum des Bandes steht die Frage, unter welchen Bedingungen an der Hochschule und darüber hinaus in den Praxisfeldern und gesellschaftlichen Räumen, in die sich die Hochschule einschreibt, diskriminierungskritisch gedacht und gehandelt werden kann. Die damit verbundenen Fragen werden aus verschiedenen Perspektiven und mit den unterschiedlichen Zugängen der Autor*innen entlang von drei thematischen Feldern bearbeitet, bewegt und weiterentwickelt. Ausgehend von einer grundlegenden Begriffsarbeit im Feld »Diversity in der Kritik« gerät die Hochschule im zweiten Feld als »machtvolle Institution« in den Blick, in 
deren Bildungsarbeit sich in einem dritten Feld »widerständige Vermittlungspraxen« entfalten.

\section{Feld I: Diversity in der Kritik}

Das erste Feld reflektiert theoretische und methodologische Diskursstränge und Ansätze, aus denen kritische Perspektiven für die Bearbeitung von Fragen zu Diversity gewonnen werden. Die Beiträge leisten einen substanziellen Beitrag zur Schärfung und Abgrenzung zentraler Begrifflichkeiten, in dem sie diese mit poststrukturalistischen, intersektionalen, postkolonialen, queeren oder rassismuskritischen Perspektiven problematisieren, präzisieren und überschreiben.

Anfang, Grundlage und Ausgangspunkt des gesamten Sammelbandes ist der Beitrag von Melissa Steyn mit Serena O. Dankwa. Dabei handelt es sich um die ins Deutsche übertragene und überarbeitete Version von Steyns wegweisendem Artikel Critical Diversity Literacy: Essentials for the 21st Century (2015). Steyn verdichtet darin ihre jahrzehntelange Auseinandersetzung mit der Thematik zu einem zehn Punkte umfassenden Konzept. In diesem entwirft sie eine Form der Literacy, welche die konstitutive Rolle von Macht in jeglichen Fragen rund um Diversität erkennt und eine vertiefte Auseinandersetzung mit dem eigenen Gewordensein innerhalb dieser Machtverhältnisse bedingt. Im Gespräch mit Melissa Steyn hat Serena O. Dankwa für die deutsche Erstübersetzung Nuancierungen und Ergänzungen vorgenommen, die für den deutschsprachigen Kontext relevant sind. So hat sie den Text etwa um aktuelle Diskurse zu Migration und Rassifizierung, Intersektionalität und dem Schweizer Asylregime erweitert.

Hiervon ausgehend unternehmen Ulla Klingovsky und Georges Pfruender den Versuch, die analytische Reichweite der Begriffe Literalität und Kritik in Critical Diversity Literacy begriffsanalytisch auszuloten. Dabei wird der Diskurs um eine legitime Literalität ebenso problematisiert wie die Frage aufgeworfen, welche Formen von Kritik den vielfältigen Verstrickungen von Hochschulbildung, Diversitätskompetenz und Wissensordnungen in aktuelle Machtverhältnisse angemessen scheint. Klingovsky und Pfruender finden schließlich in der Taktik des Queerens ein Instrument, mit dem die Künstlichkeit der routinisiert und selbstverständlich scheinenden, stets machtvollen Verfahren, Abläufe und Mechanismen an der Institution Hochschule aufgespürt und aufgestört werden können.

Eine fundamentale Kritik am Begriff Diversity entwickelt Stefanie Claudine Boulila ausgehend von der Frage, ob und inwiefern der Diskurs um Vielfalt und Diversität antirassistischen Ansprüchen gerecht werden kann. Sie kritisiert, dass in diesen Diskursen Antirassialismus ahistorisch und machtunkritisch normalisiert wird. Darüber hinaus veranschaulicht sie, inwiefern der Begriff Diversity ohne Bezug zu einer sozialen Bewegung, die gegen strukturelle Ungleichheiten ankämpft, in der Gefahr steht, den strukturellen Charakter von Rassismus zu 
dethematisieren, Differenzierungsprozesse weiterhin zu verdecken und machtkritische Analysen zu blockieren. Boulila problematisiert, dass sich Institutionen zwar nach außen hin zu Diversity bekennen, diese aber real nicht umsetzen, sondern sich damit primär gegen den Vorwurf des Rassismus schützen wollen und so offene Auseinandersetzungen mit Rassismus verstummen lassen. Gleichzeitig deutet Boulila die Möglichkeit an, Diversity als eine Art Trojanisches Pferd strategisch einzusetzen.

Lucienne Wagner kritisiert in ihrem Beitrag ebenfalls den Begriff Diversity. Aus postkolonialer und queerer Perspektive beschäftigt sie sich mit dem Problem, dass der Begriff im Rahmen einer fortschreitenden Neoliberalisierung der Hochschule weniger zu einer solidarischen, horizontal und breit aufgestellten Antidiskriminierungspolitik, sondern stattdessen zu einer Ressourcenverknappung in der Gleichstellungsarbeit führt. Zugleich eröffnet sie die Denkmöglichkeit, den unscharfen Begriff Diversity - trotz und gerade wegen seiner Uneindeutigkeit als Spannungsbegriff produktiv einzusetzen. Dies bedingt jedoch, dass DiversityPraktiker*innen der Depolitisierung von Diversity mittels kritischer Ansätze aktiv entgegenwirken. Im Anschluss an die Initiativen studentischer und antirassistischer Kollektive sowie queerer und postkolonialer Theorien zeigt sie, dass es bei der Dekolonisierung von Universitäten nicht nur um eine Diversity of People gehen kann, sondern dass vielmehr auch eine Diversity of Knowledges gefordert ist.

In diesem Sinne schließt María do Mar Castro Varela mit ihrem Text den Kreis um das erste Feld »Diversity in der Kritik«. Ausgangspunkt ihres Beitrags sind die auf eine affirmative Sabotage zielenden pädagogischen Bemühungen von Gayatri Chakravorty Spivak. Im Anschluss an eine differenzierte Auseinandersetzung mit der breit rezipierten Spivak'schen Konzeption des Verlernens stellt Castro Varela ihr Konzept der kontrapunktischen Bildung als Entwurf einer (Bildungs-)Praxis vor, in der die Vorstellung eines »monolithischen Wissens « verabschiedet wird. Die kontrapunktische Bildungsarbeit zielt auf eine Critical Literacy, die als affirmative Sabotage spiralförmig gegen eine imperiale Zugerichtetheit interveniert und darin eine immer wiederkehrende vertiefte und kritische Analyse des eurozentrischen Wissenskanons evoziert. Die Überlegungen münden - kontrapunktisch - in ein Kompositionsprinzip polyphoner Mehrstimmigkeit, in der sich die vielen Gegenstimmen artikulieren und mit den (dominanten) Leitstimmen in ein Konzert eintreten, in dem Wissensbestände und Epistemologien als umkämpftes Terrain hörbar werden.

\section{Feld II: Die Hochschule als machtvolle Institution}

Im Anschluss an diese grundlegende Arbeit an den Begriffen beleuchtet das zweite Feld die gesellschaftlichen Verstrickungen, die in der Hochschule wirken. Aus unterschiedlichen Perspektiven werden hier die historisch gewachsenen Ungleich- 
heitsverhältnisse und Wechselwirkungen zwischen privilegierten und diskriminierten gesellschaftlichen Positionen thematisiert, welche die institutionalisierten Praxen der Hochschule prägen. Darüber hinaus werden diese Praxen selbst als ein Schauplatz in den Blick genommen, an dem Macht- und Ungleichheitsverhältnisse gestützt und aufrechterhalten werden.

Mit Blick auf die Hochschule und ihr Verhältnis zu institutionellem Rassismus und intersektionalen Ausschlussmechanismen eröffnet Vanessa E. Thompson die Auseinandersetzungen in diesem zweiten Feld. Der Beitrag veranschaulicht die historische Kontinuität kolonialer Machtverhältnisse an der Hochschule und hinterfragt das Potenzial von Diversitätspolitiken in der unternehmerischen Hochschule grundlegend. Bezüglich der Reproduktionsformen und Wirkweisen von Rassismus an der Hochschule analysiert die Autorin, auf welche Weise DiversityMainstreaming und Gleichstellungspolitiken ihr Ziel, einen substanziellen Beitrag $\mathrm{zu}$ Antidiskriminierung an der Hochschule zu leisten, systematisch verfehlen. Am Ende ihres Beitrags skizziert sie Abolitionismus als eine Perspektive, mit der Räume des organisierten Widerstands, der Subversion und der Akkumulation von Wissen zu nicht-kapitalistischen Zwecken denkbar werden. Hierbei scheint eine gleichzeitige Stärkung und Dekolonialisierung der Hochschule nur über ihre Abschaffung im Sinne einer wesentlichen Transformation möglich.

Nathalie Amstutz analysiert das Diversitätswissen und dessen Kritik an Hochschulen aus einer wirtschaftswissenschaftlichen Perspektive. Auf der Basis der analytischen Kategorien des Neoinstitutionalismus rekonstruiert der Beitrag die Entstehung und Ausbreitung von Rationalitätsmythen, mit denen Organisationen die Professionalität ihrer Programme und bürokratischen Strukturen ausweisen und legitimieren. Die Autorin argumentiert, dass hinter einer derartigen Legitimationsfassade Fragen von Gleichstellung lediglich oberflächlich bearbeitet, tieferliegende strukturelle Zusammenhänge hingegen verdeckt werden. Die Perspektive der Organisationsforschung zeigt darüber hinaus deutlich, dass der für Hochschulen maßgebliche individuelle Leistungsdiskurs eine kritische institutionelle, strukturelle und politische Auseinandersetzung mit Fragen von Diversität erschwert.

Ausgangspunkt der Studie, die Maritza Le Breton und Susanne Burren in ihrem Beitrag vorstellen, bildet der Internationalisierungsimperativ schweizerischer Fachhochschulen. Vor diesem Hintergrund stellt die Studie die Frage, inwiefern sich im Zuge der Internationalisierung für migrantische Studierende neue Zugehörigkeitserfahrungen und/oder neue Ungleichheiten aufgrund von Geschlecht und Migration herausbilden. Die Autorinnen machen deutlich, dass die Internationalisierung an Hochschulen oftmals im Sinne einer ökonomischen Ressource gefördert wird. Dabei bleibt der Umstand, dass im Zeichen der Internationalisierung vorhandene Ungleichheiten intensiviert und bestehende Machtverhältnisse 
reproduziert werden, weitgehend unthematisiert - eine Forschungslücke, der Le Breton und Burren in diesem Forschungsbericht nachgehen.

Brian McGowan und Herbert Bichsel beleuchten die Praxen der Hochschule aus ableismuskritischer Perspektive und verhandeln die Situation von Studierenden mit Behinderungen an Schweizer Hochschulen. Bei ihrer Analyse rekurrieren sie auf den Begriff des "Aussortiertwerdens", der es ihnen ermöglicht, sowohl institutionelle und personenbezogene Machtstrukturen als auch die historische Gegenwärtigkeit von Unterdrückungs- und Diskriminierungsstrukturen sichtbar zu machen. Als zweites Analyseinstrument dient ihnen das Übereinkommen über die Rechte von Menschen mit Behinderung (UN-BRK) als Richtschnur dafür, wie eine vollumfängliche Partizipation von Menschen mit Behinderung aussehen könnte. Im Anschluss an ihre Analyse fordern die beiden Autoren nicht weniger als eine radikale Erneuerung des heutigen Bildungssystems und eine Erweiterung des Bildungsbegriffs, verstanden als Schutz und Förderung der Menschenrechte.

Natalie Kofel Berger verdichtet in ihrer verschriftlichten Rede spezifische Thesen zum Thema Inklusion in fünf programmatisch-poetischen Sätzen. Zunächst imaginiert sie anhand des Konzepts »Universal Design« eine Sprache und Form, die für unterschiedliche Disziplinen zugänglich wäre. Ausgehend von den Möglichkeiten der Kunst beschreibt sie Emotionen, insbesondere Angst (kombiniert mit Neid) vor dem »Anderen « und die daraus resultierenden Abwehrreaktionen als zentrale behindernde Faktoren. Auf der Suche nach einer Bildung, die zur Überwindung der Angst beitragen könnte, gelangt die Autorin zu Patrice Khan-Cullors Metapher des Sternenstaubs, wonach menschliche Individuen immer auch Teil eines magischen großen Ganzen sind (vgl. Khan-Cullors 2020: 15). Der Beitrag erinnert an die Rolle von Gefühlen und die Bedeutung unser aller emotionaler Involviertheiten in die scheinbar unüberwindbaren Gegensätze zwischen In- und Exkludierten. Daher, so folgert die Autorin, braucht es nicht nur ein kritisches Bewusstsein zum Erkennen der relevanten Machtdimensionen, sondern auch eine Art der Liebe als Gegenmittel zur Angst und Sinn für die Verbundenheit, die uns dazu befähigt, gemeinsam die notwendigen Energien für einen strukturellen und kulturellen Wandel zu entwickeln.

Das Feld »Die Hochschule als machtvolle Institution« schließend, reflektieren Jonah Garde und Simon Harder im dialogischen Prinzip Ansätze, Potenziale und Herausforderungen trans" formativer Pädagogik. Als eine intersektional und transdisziplinär verstandene Praxis soll trans"formative Pädagogik normativ gesicherte Bereiche des Schweigens unterbrechen und Gebiete der Unsprechbarkeit hervorbringen. Trans" formative Pädagogik will an den Grenzen dominanter anerkannter und legitimierter Formen von Wissensproduktion ansetzen und als eine verkörperte Vermittlungspraxis ein Bewusstsein dafür hervorbringen, dass Körper und körperliches Erleben Voraussetzung für ein tieferes Verständnis der individuellen und kollektiven Situiertheit in Macht- und Gewaltverhältnissen sind. Garde und 
Harder plädieren für ein Dezentrieren von Weiß-Sein und für ein Verschieben des Analysefokus hin zu vielfältigeren Zielgruppen. Dies mit dem Ziel, ein kollektives Verständnis dafür zu entwickeln, wie jede*r in diese Transformationsprozesse eingebunden ist.

\section{Feld III: Widerständige Vermittlungspraxen}

Das dritte Feld zielt schließlich auf eine kritische Auseinandersetzung mit der eigenen Disziplin und deren impliziten Normalitäts- und Wissensordnungen. Dabei kommen die Zusammenhänge zwischen der Hochschule und ihren jeweiligen Praxisfeldern (bzw. ihren gesellschaftlichen Bezugsräumen) sowie die eigene Praxis des Lehrens, Lernens, Forschens, Planens, Beratens und/oder Organisierens in den Blick.

Den Auftakt in dieses Feld macht der Beitrag von Karolin Heckemeyer, der sich mit den Konstruktionen von un_sportlichen Körpern im Sport- und Bewegungsunterricht beschäftigt. Die Autor*in widmet sich der Frage, wie und auf welcher Grundlage Körper im Kontext der Lehrpersonenbildung im Fach Bewegung und Sport als Anker für soziale Zuschreibungen von Un_Sportlichkeit und/oder In_Kompetenz positioniert werden. Heckemeyer interessiert sich nicht nur für die Frage, was die Dozierenden des Faches selbst verkörpern, sondern darüber hinaus für die ethnisierten, vergeschlechtlichten und ableisierten Körperbilder von Studierenden, vor deren Hintergrund sich diese als potenzielle Sportlehrpersonen entwerfen. Der Beitrag zeigt die Notwendigkeit, vorhandene stereotype Körperbilder und deren Entstehungskontexte in der Hochschullehre explizit zu thematisieren und $\mathrm{zu}$ dekonstruieren als eine Bedingung dafür, dass angehende Sport- und Bewegungspädagog*innen eine diversitätsorientierte Lehrpraxis gestalten können.

Simon Affolter und Vera Sperisen fragen ebenfalls, was an Hochschulen thematisiert und dekonstruiert werde müsste, um rassismuskritische Bildung an Schulen zu ermöglichen. Dies reflektieren sie im Anschluss an ihr Forschungsprojekt »Doing/Undoing Difference in der politischen Bildung « mit den Teilnehmenden des Projekts. Während sowohl für die Lehrpersonen als auch die Schüler*innen unbestritten ist, dass Migration und Flucht ein aktuelles und bedeutsames gesellschaftliches Themenfeld darstellen, das im Unterricht behandelt werden soll, erleben beide Gruppen die Unterrichtspraxis als unbefriedigend. Affolter und Sperisen fragen nach möglichen Gründen, weshalb wegweisende Ansätze aus der Migrations- und Bildungsforschung in der pädagogischen Praxis bislang kaum Resonanz erzeugt haben.

Ausgehend von seiner eigenen Bildungserfahrung in der Auseinandersetzung mit Autor*innen der Postmoderne stellt Jörg Wiesel die Frage, wie das postmoderne Denken und dessen Begriff von Kritik heute an Studierende als eine performative 
Praxis vermittelt werden können. Er sieht in der Analyse der Postmoderne - eine soziale und kulturelle Haltung, die eine radikale Pluralität als Grundverfassung der Gesellschaften anerkennt - das Potenzial für eine bildende Auseinandersetzung mitaktuellen Themen von Vielfalt und Differenz. Diese pädagogischen Überlegungen illustriert er anhand von zwei Beispielen aus unterschiedlichen Kontexten. Einerseits ist dies die Beschäftigungmit einer Filibuster-Rede, die er in der Tradition feministischer Performance-Kunst einordnet, andererseits mit der Methode des lauten Lesens. Beide Beispiele versteht Wiesel letztlich als Elemente einer performativen Auseinandersetzung mit Critical Diversity Literacy, die dazu beitragen soll, das Überschreiten von Identitäten zu ermöglichen.

Nina Mühlemann eröffnet eine gänzlich neue Facette, indem sie sich auf Margaret Prices Theorie der Crip Spacetime (2016) bezieht und im Anschluss daran fordert, dass räumliche und zeitliche Gegebenheiten neu zusammen gedacht werden müssen. Sie beginnt ihren Text mit Fragen von Zugang und Zugänglichkeit und zeigt deren emotionale und körperliche Konsequenzen auf. Daran anschließend konturiert sie ein Verständnis von Zugänglichkeit als Teil der Disability Aesthetics, der immer wieder auf spezifische Situationen hin anzupassen ist. Aus der Perspektive ihrer Arbeit als Theatermacherin und forschende Performerin macht Mühlemann sichtbar, wie Fragen der Präsenz von Behinderung stets Probleme und Widersprüche verursachen, die wiederum als Irritationen neues Wissen und neue Erfahrungen produzieren.

Die Produktion von neuem Wissen und das Teilen neuer Erfahrungen ist auch das Ziel des Resonanzraums, den Alisha M.B. Heinemann und Berna Keser eröffnen. Dieser Resonanzraum entsteht durch das Verweben von vielfältigen und komplexen Stimmen von Lehrenden und Studierenden of Colour. Diese Stimmen begeben sich in einen fiktiven Dialog miteinander und mit rassismuskritischen Konzepten wie den »Practices of Silencing« (Dotson 2011; Küçükgöl 2019), »Space invaders« (Puwar 2004; Settles et al. 2018), »The feminist killjoy« (Ahmed 2010) und »The margin as place for radical openness« (hooks 1993). Mit diesem experimentellen Zugang des fiktiven Gesprächs werden einerseits marginalisierte Stimmen und Praxen hör- bzw. lesbar, andererseits fügen Heinemann und Keser auch der Critical Diversity Literacy eine weitere Dimension hinzu. Damit initiieren sie eine transformatorische Praxis innerhalb akademischer Räume, die das Lehren und Lernen im Hochschulraum von den Rändern her zu verändern vermag.

Eine kritisch-performative Lektürepraxis, die auf eine Analyse, Problematisierung und Veränderung der gegebenen Verhältnisse drängt, wird von den hier versammelten Beiträgen durchgängig als Zielperspektive für die zukünftige Bearbeitung von Fragen zu Diversität interpretiert. Sie gilt - wie bereits erwähnt - eher als Leitbild einer erst zu entwickelnden Hochschulpraxis denn als Beschreibung des Status quo. Unstrittig scheint weiterhin, dass das Projekt einer Critical Diversity Literacy im Hochschulraum von vielfältigen Bezugstheorien, von anderen Refe- 
renzautor*innen und bislang ungehörtem Wissen profitiert. Vielleicht könnte auf diese Weise dem vielfach geäußerten Verdacht begegnet werden, dass DiversityBegriffe, -Konzepte und -Politiken sich lediglich als neue Gesten der Leistungsnorm und als Ausdruck einer raffinierten akademischen Überbietungsstrategie erweisen könnten. Wenn die politischen Dimensionen wissenschaftlicher Fragen, aktivistischer Gegenbewegungen und praktischer Bildungsarbeit nicht ausgeklammert, sondern von den Rändern ins Zentrum gerückt werden können, öffnet sich möglicherweise der Raum für neue Imaginationen sozialer und institutioneller Wirklichkeiten.

\section{Literatur}

Ahmed, Sara (2010): The promise of happiness, Durham, NC: Duke University Press.

Bratič, Lubomir (2012): Politischer Antirassismus, Wien: Löcker.

Crenshaw, Kimberlé/Gotanda, Neil/Peller, Gary/Thomas, Kendall (1995): Critical Race Theory: the key writings that formed the movement, New York: The New Press.

Crenshaw, Kimberlé (1989): »Demarginalizing the Intersection of Race and Sex. A Black Feminist Critique of the Rhetoric of Solidarity and Betrayal«, in: Anne Phillips, Feminism and Politics, Oxford: Oxford University Press, S. 314-343.

Dotson, Kristie (2011): »Tracking Epistemic Violence, Tracking Practices of Silencing «, in: Hypatia 26 (2), S. 236-257, www.jstor.org/stable/23016544 [15.3.2021].

Eggers, Maisha/Kiloma, Grada/Pesche, Peggy/Arndt, Susan (2005): Mythen, Masken und Subjekte: Kritische Weißseinsforschung in Deutschland, Münster: Unrast.

hooks, bell (2006 [1993]): Yearning. Race, gender, and cultural politics. 13th print, Boston, MA: South End Press.

hooks, bell (1990): »Schwesterlichkeit: Politische Solidarität unter Frauen«, in: beiträge zur feministischen theorie und praxis, 13. Jg., Heft 27, 1990, S. 77-92.

Khan-Cullors, Patrisse (2020): \#BLACK LIVES MATTER. Eine Geschichte vom Überleben, Köln: Kiepenheuer \& Witsch.

Küçükgöl, Dudu (2019): "Spricht sie noch oder schweigt sie schon? SilencingStrategien gegen muslimische Frauen in der feministischen Praxis«, in: Alisha M. B. Heinemann/Natascha Khakpour (Hg.), Pädagogik sprechen. Die sprachliche Reproduktion gewaltvoller Ordnungen in der Migrationsgesellschaft, Stuttgart: J.B. Metzler S. 83-98.

Mecheril, Paul/Klingler, Birte (2010): »Universität als transgressive Lebensform. Anmerkungen, die gesellschaftliche Differenz- und Ungleichheitsverhältnisse berücksichtigen«, in: Lucyna Darowska/Thomas Lüttenberg/Claudia Machold 
(Hg.), Hochschule als transkultureller Raum? Kultur, Bildung und Differenz in der Universität. Bielefeld: transcript; 2010, S. 83-117.

Mills, Charles W. (1997): The Racial Contract, Ithaca/New York: Cornell University Press.

Pratt, Marie Luise (1996): Apocalypse in the Andes: Contact Zones and the Struggle for Interpretive Power, Encuentros 15. Washington: IDB Cultural Center, http: //idbdocs.iadb.org/wsdocs/getdocument.aspx?docnum=1774431 [15.3.2021].

Price, Margaret (2016): »Un/Shared Spaces«, in: Jos Boys (Hg.), Disability, Space, Architecture. London/New York: Routledge, S. 155-172.

Meyer, Katrin/Purtschert, Patricia (2010): »Die Macht der Kategorien. Kritische Überlegungen zur Intersektionalität«, in Feministische Studien, 28 (1), S. 130142.

Puwar, Nirmal (2004): Space invaders. Race, gender and bodies out of place, Oxford/New York: Berg.

Settles, Isis H./Buchanan, NiCole T./Dotson, Kristie (2018): »Scrutinized but not recognized: (In)visibility and hypervisibility experiences of faculty of color «, in: Journal of Vocational Behavior 113, S. 62-74. DOI: 10.1016/j.jvb.2018.06.003 [15.3.2021].

Sternfeld, Nora (2013): Kontaktzonen der Geschichtsvermittlung: Transnationales Lernen über den Holocaust in der postnazistischen Migrationsgesellschaft, Wien: Zaglossus.

Steyn, Melissa (2015): »Critical Diversity Literacy: Essentials for the twenty-first century«, in: Vertovec, Steven (Hg.), Routledge International Handbook of Diversity Studies, London/New York: Routledge, S. 379-389. 
